\title{
VALIDACIÓN DE UN INSTRUMENTO PARA DETECTAR SÍNTOMAS INDICATIVOS DE CÁNCER DE OVARIO EN QUERÉTARO, MÉXICO.
}

\section{Autores:}

Cabrera Sánchez, Rosalina ${ }^{1}$

Juárez Lira, Alberto ${ }^{2}$

Gallegos-Torres, Ruth ${ }^{2}$

Álvarez-Aguirre, Alicia ${ }^{2}$

López-Sánchez, Nepthys ${ }^{2}$

Sánchez-Peralta, Mercedes ${ }^{2}$

1 Especialidad en Salud pública, Facultad de enfermería, Universidad Autónoma de Querétaro (UAQ), México.

${ }^{2}$ Doctor en Ciencias de la salud, Jefe de investigación y posgrado de la Facultad de Enfermería, Univ. Autónoma de Querétaro (UAQ), México.

2 Doctora en Ciencias de la salud, Docente de investigación de la Facultad de Enfermería, Univ. Autónoma de Querétaro (UAQ), México.

2 Doctora en ciencias de la Enfermería, Docente de investigación de la Facultad de Enfermería, Univ. Autónoma de Querétaro (UAQ), México.

2 Doctora en Ciencias de la salud, Docente de investigación de la Facultad de Enfermería, Univ. Autónoma de Querétaro (UAQ), México.

2 M.C. Enfermería, Docente de investigación de la Facultad de Enfermería, Universidad Autónoma de Querétaro (UAQ), México.

Correspondencia: alberto.juarezlira@gmail.com 


\section{RESUMEN}

El objetivo de este trabajo fue validar un instrumento para detectar a mujeres con riesgo de padecer Cáncer de Ovario (CaOva).

Material y Métodos: se realizó una búsqueda exhaustiva de datos que revelen síntomas indicativos del CaOva, dicha búsqueda se refiere a los principales síntomas sugestivos y con ello se realizó un entrevista estructurada, en la colecta de datos se analizaron palabras claves y con ello se formularon preguntas concernientes a los antecedentes, síntomas y duración; también dolor y/o inflamación abdominal/pélvico, frecuencia urinaria y saciedad temprana, así como antecedentes familiares y propios; se realizo un prueba piloto a 100 mujeres en UNEME-DEDICAM.

Resultados preliminares: La evaluación de la consistencia interna fue de KR-20 $=0,19$. Se obtuvo que el $2 \%$ presento saciedad temprana, $1 \%$ dolor abdominal, 17\% tuvo antecedente de Cáncer familiar, 7\% antecedente propio.

Conclusiones: El instrumento empleado funciona como método de tamizaje y puede complementarse con pruebas de laboratorio y gabinete. Los resultados obtenidos concuerdan con los síntomas referidos por el colegio Mexicano de especialistas en ginecológica y obstetricia (COMEGO).

Palabras clave: Cáncer de ovario, Tamizaje masivo, prevención secundaria. 


\section{ABSTRACT}

The objective of this research was an instrument to detect women with risk of obtaining ovarian cancer (CaOva).

Materials and Methods: an exhaustive search was conducted to collect data in order to show symptoms from CaOva, that research it means to principal suggestive symptoms and with that it realized an unstructured interview, in data collect were analyzed key words and with that questions were asked about the background, symptoms and length; also pain and abdominal pain, pelvic pain, urinary regularity, early satiety and medical background family and individual. A pilot test was conducted to 100 women on UNEME-DEDICAM.

Preliminary Results: The evaluation of the consistent intern were as follow: KR-20=0,19. $2 \%$ presented with early relief, $1 \%$ abdominal pain, $17 \%$ had a history of family cancer, $7 \%$ were own history. Conclusion: The instrument used works as screening method with laboratory test and cabinet. The results agree with the symptom reported by Mexican school of specialist in gynecology and obstetrics (COMEGO).

Keywords: Ovarian Cancer, mass screening, screening prevention. 


\section{INTRODUCCIÓN}

El Cáncer de ovario (CaOva) se posiciona como la tercera neoplasia ginecológica a nivel mundial. En México es la cuarta causa de muerte por cáncer entre la población femenina ${ }^{(1)}$, siendo su incidencia de 5.6 mujeres afectadas por cada 100 mil habitantes (2); a pesar que se cuenta con datos estadísticos de importancia falta de métodos de cribado en el diagnostico de Cáncer de Ovario (CaOva) han retrasado que se atienda de manera oportuna dicha patología.

Hoy en día existe diversa información que nos habla sobre los factores protectores y de riesgo que una mujer debe de conocer para evitar desarrollar CaOva entre los que podemos encontrar que hay factores dietéticos específicos como el consumo de grasas saturadas ${ }^{(3)}$, estilos de vida como lo es vivir en constante estrés y el trabajar de noche ${ }^{(4-5)}$ y gineco-obstétricos como el haber cursado con endometriosis y tener denso tejido mamario ${ }^{(6-7)}$ que se asocian consistentemente con el riesgo de CaOva. De igual manera existen factores a los que se les ha asociado con la prevención o reducción del riesgo a desarrollar CaOva entre ellos farmacológicos haciendo referencia al consumo de Aspirina o metformina ${ }^{(8-9)}$, antecedentes y cirugías gineco-obstetricos específicamente la ligadura de trompas e histerectomía ${ }^{(10)}$, los hábitos alimenticios $^{(11)}$ y vacunas ${ }^{(12)}$.

Estudios recientes, así como diversos autores sugieren que al identificar a las mujeres con síntomas indicativos de CaOva, mas el uso de biomarcador como el CA 125 y el Ultrasonido transvaginal (USTV) ${ }^{(13)}$ pueden establecerse 
como método masivo de detección. Los síntomas suelen estar presentes en etapas tempranas y tardías lo cual permite su identificación oportuna (14). Basados en guías clínicas como la de el Institute for health and clinical excellence (NICE), Ministerio de salud (AUGE), Sociedad médica española (ESMO), Instituto nacional de enfermedades oncológicas, Instituto nacional de cancerología (INCAN), que informan sobre los síntomas presentes en las mujeres. Con la finalidad de identificar estos síntomas estudios anteriores hacen referencia a que se debe preguntar la frecuencia con que estos se presentan ${ }^{(15-18)}$. Con el coste de los actuales métodos de diagnostico y con la necesidad de desarrollar una herramienta especifica que nos funcione como método de tamizaje para identificar a mujeres con riesgo y aplicarles las pruebas pertinente se propone el instrumento "Diagnostico Oportuno de Cáncer de Ovario".

\section{MATERIAL Y MÉTODOS}

Se realizó la validación de un instrumento para la detección oportuna de cáncer de ovario basado en síntomas indicativos ya establecidos por diferentes autores expertos en el tema. Hablamos de validación, cuando queremos aprobar algo que nos sea de ayuda, para la recolección de datos ya sea por medio de entrevistas o cuestionarios ${ }^{(19)}$.

Procedimiento

Paso 1. Se realizó una búsqueda exhaustiva en las bases de datos Bvs, Pubmed, Lilacs y Scielo de los factores y síntomas utilizando el término Ovarian Neoplasms, priorizando según la frecuencia con que se presentó cada uno de ellos (anexo I). Paso 2. Con lo anterior se dió paso a formular una serie 
de preguntas conformadas por 6 ítems, con respuestas de Si y No que para considerarse positivas tuvieron que cumplir lo siguiente ser un síntoma real que se produjera 13 días o más al mes y estuviera presente al menos 6 meses y menos de 1 año; fueron aplicadas a un grupo de 9 estudiantes de nivel posgrado de diferentes disciplinas con el fin de buscar si los términos utilizados eran adecuados y comprensibles. Paso 3. Se agregaron datos sociodemográficos (nombre, edad, domicilio, derechohabiencia, curp, fecha y entidad de nacimiento), instrucciones y una explicación detallada para que las participantes supieran que hacer $\mathrm{o}$ a donde se van a dirigir ante resultados positivos. Paso 4. Se solicitó el permiso de la Unidad de Especialidades Medicas Dedicada a la Detección y Diagnostico del Cáncer de Mama (UNEME-DEDICAM) ubicada en el municipio de Santiago de Querétaro, México, donde se realiza una primera aplicación en Mayo del 2015 de los 6 ítems a un grupo de 30 mujeres que en ese momento se encontraban en la unidad quienes fueron invitadas a participar con el fin de obtener información sobre si los ítems eran comprensibles, si entendían el carácter de cada pregunta, si el tiempo era el adecuado o si existía rechazo por alguna pregunta, con ello se comprobó que el instrumento era aceptado y comprendido por la población femenina. Paso 5. En una segunda aplicación se estimó la población de estudio que es de un número de 15147 mujeres residentes del estado de Querétaro, con una frecuencia esperada de $5.6 \%$, un límite de confianza del $5 \%$, un efecto del diseño de 1.0 y un cluster de 1 con un Intervalo de confianza de $95 \%$ obtenidos a través del programa de cálculo de EPI info 7, obteniéndose así una muestra de 100 mujeres. Se invitó a participar a toda mujer que se encontraba en ese momento en la UNEME-DEDICAM, que cumpliera con los 
criterios de inclusión establecidos de estar en un rango de edad entre 50 y 69 años, que no contara con oferectomia total previa, estuviera de acuerdo y firmara el consentimiento informado.

\section{Recolección de datos}

Los datos se obtuvieron a través de preguntas directas por el investigador principal. El trabajo de campo se realizo el mes de Mayo de 2015. Se solicitó consentimiento por escrito a todas las participantes y se obtuvo la Autorización del programa estatal de cáncer en la mujer de los Servicios de Salud del Estado de Querétaro (SESEQ).

\section{Análisis de la información}

Para el análisis de la información se concentró la información en el software estadístico IBM SPSSv.21; se realizó análisis univariado por medio de frecuencias y porcentajes, análisis de la consistencia interna utilizando KR-20; Con una sensibilidad de $81.48 \%$, especificidad del $89.04 \%$, un valor predictivo positivo de $73.33 \%$ y un valor predictivo negativo $92.85 \%$, con un falso positivo igual a $10.95 \%$ y un falso negativo de $18.51 \%$.

\section{RESULTADOS}

En el presente estudio realizado en el periodo de Enero a octubre del 2015 se conto con la participación de 100 mujeres; se observó que de acuerdo a los antecedentes y síntomas no cumplen con las condiciones 73 (73\%), aquellas que tuvieron antecedente de cáncer familiar representan el 17 (17\%) 
y antecedente propio 7 (7\%), y con síntoma de saciedad temprana el 2 (2\%) y dolor abdominal 1 (1\%). El promedio de edad fue de 56.7 años; encontrándose los grupos de edad más afectados entre los 50 y 59 años, seguido de los 6069 , con el $70(70 \%)$ y el $30(30 \%)$ respectivamente (figura I).

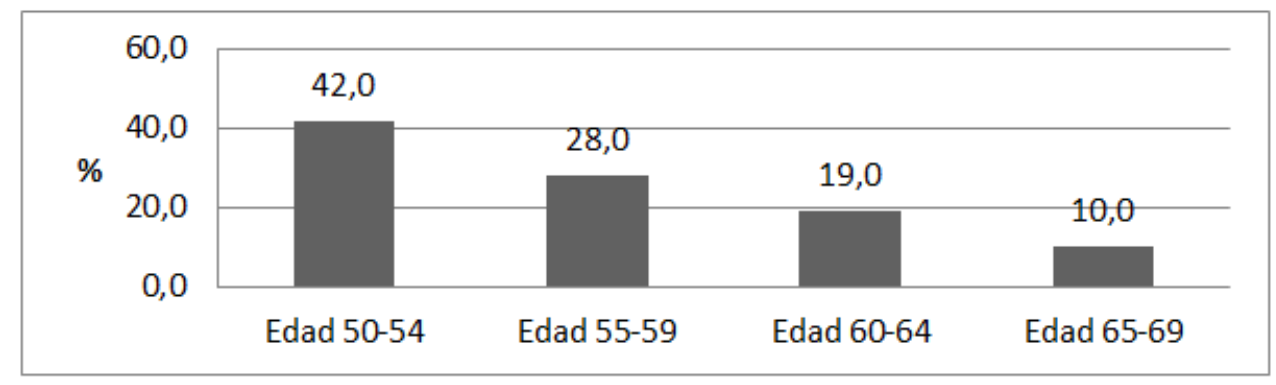

FIGURA I. FRECUENCIA DE LA EDAD EN MUJERES PARTICIPANTES. QUERETARO 2015

Fuente: Instrumento Diagnóstico Oportuno de Cáncer de Ovario 2015

En base a los resultados de la herramienta "Diagnostico oportuno de cáncer de Ovario" con respecto a las edades de un total de 27 positivos se obtuvo que el $12 \%$ se encontraba en la edad de 50 a 54 años, el $10(10 \%)$ en una edad de 55 a 59 años, el $3 \%$ tenia de 60 a 64 años y el $2(2 \%)$ estuvo en la edad de 65 a 69 años (figura II).

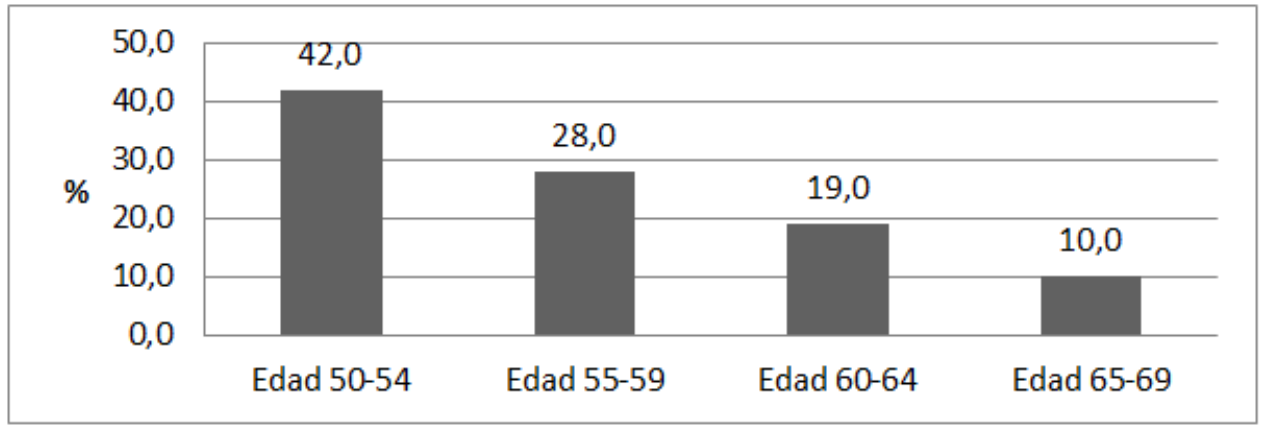

FIGURA II. FRECUENCIA DE SÍNTOMAS POSITIVOS CON RESPECTO A LA EDAD DE LAS PARTICIPANTES. QUERETARO 2015.

Fuente: Instrumento Diagnóstico Oportuno de Cáncer de Ovario 2015 
Con una consistencia interna de KR- $20=0,19$ se observa que la obtenida en el instrumento es muy baja, por lo que se necesita realizar una reestructuración tomando como referencia que se requieren de 2 o más síntomas para considerarse positivo $(k R-20=0,78)$ con ello se puede asegurar un KR-20 mayor.

\section{DISCUSIÓN}

El proceso de validación implica una serie de pasos a seguir con el objetivos de aprobar que algo que nos es de ayuda para la recolección de datos atravesé de entrevistas y cuestionarios.

En el presente trabajo, el instrumento propuesto "Diagnostico oportuno de Cáncer de Ovario" trata de identificar a las mujeres con síntomas que se produzcan 13 días en 1 mes y más de 6 meses en 1 año dando un resultado de 27 casos positivos complementados por una serie de estudios de laboratorio y gabinete (CA-125 y USTV) que en conjunto llevan al diagnostico oportuno de cáncer de ovario; los síntomas presentados obtuvieron la siguiente puntuación, el $1 \%$ presento dolor abdominal el $2 \%$ saciedad temprana al comer en una muestra de 100, que concuerdan con los síntomas referidos por el colegio mexicano de especialistas en ginecologia y obstetricia ${ }^{(20)}$ (COMEGO) los cuales son saciedad temprana y dolor abdominal.

De acuerdo con el National Cáncer Institute ${ }^{(21)}$ (NIH) el antecedente familiar de cáncer en primera línea es un gran factor de riesgo para cáncer de 
ovario, y de acuerdo con los casos positivos arrojados el $17 \%$ presentó este factor con respecto a los demás síntomas.

De acuerdo al Globocan ${ }^{(22)}$ el cáncer de ovario ocupa el $4.0 \%$ de todas las patologías oncológicas en la mujer. El $12 \%$ de las participantes con síntomas positivos se encontraban en un rango de edad de 50 a 54 años que de acuerdo con lo estipulado en el tercer consenso nacional de cáncer de ovario 2011 el rango de edad en el que incide mayormente se encuentra entre 50 a 70 años.

\section{AGRADECIMIENTOS}

Este trabajo recibió el apoyo financiero del Consejo Nacional de Ciencia y Tecnología (CONACYT) a través del Programa Nacional de Posgrados de Calidad (PNPC). Los autores agradecen a las personas e instituciones que hicieron posible este estudio; Declaración de conflicto de intereses: Los autores declararon no tener conflicto de intereses. 


\section{REFERENCIAS BIBLIOGRÁFICAS}

1. Dolores Gallardo, Cantú D, Alanis P, Alvarez MA, Bañuelos J, et., al. Tercer conceso nacional de cáncer de ovario 2011 Grupo de Investigación en Cáncer de ovario y tumores ginecológicos de México "GICOM" [Internet]. Revista de Investigación Clínica; 2011. Avaladle from: http://www.gicom.org.mx/pdf/3erCONSENSONACIONALCANCERDEOVARIO.pdf

2. World Healt Organization. Globocan 2012: Estimated Cancer Incidence, Mortality and Prevalence Worldwide in 2012. [Internet]. Available from: http://globocan.iarc.fr/Pages/fact_sheets_population.aspx

3. Merritt MA, Cramer DW, Missmer SA, Vitonis AF, Titus LJ, Terry KL. Dietary fat intake and risk of epithelial ovarian cancer by tumour histology. $\mathrm{Br} \mathrm{J}$ Cancer. 2014 Mar 4;110(5):1392-401.

4. Gao. Chronic stress promoted the growth of ovarian carcinoma via increasing serum levels of norepinephrine and interleukin-10 and altering nm23 and NDRG1 expression in tumor tissues in nude mice. Biosci Trends [Internet]. 2013 [cited 2015 Oct 5]; Available from: http://www.biosciencetrends.com/getabstract.php?id=641

5. Bhatti $P$, Cushing-Haugen $K L$, Wicklund $K G$, Doherty JA, Rossing $M A$. Nightshift work and risk of ovarian cancer. Occup Environ Med. $2013 \mathrm{Apr}$ $1 ; 70(4): 231-7$.

6. Borghese B, Santulli P, Vaiman D, Alexandre J, Goldwasser F, Chapron C. Les cancers de l'ovaire associés à l'endométriose: physiopathologie et conséquences sur la pratique clinique. J Gynécologie Obstétrique Biol Reprod. 2013 Jun;42(4):325-33.

7. Wernli KJ, O'Meara ES, Kerlikowske K, Miglioretti DL, Muller CY, Onega T, et al. Investigation of Mammographic Breast Density as a Risk Factor for Ovarian Cancer. J Natl Cancer Inst. 2014 Jan 1;106(1):djt341.

8. Trabert B, Ness RB, Lo-Ciganic W-H, Murphy MA, Goode EL, Poole EM, et al. Aspirin, Nonaspirin Nonsteroidal Anti-inflammatory Drug, and 
Acetaminophen Use and Risk of Invasive Epithelial Ovarian Cancer: A Pooled Analysis in the Ovarian Cancer Association Consortium. J Natl Cancer Inst. 2014 Feb 1;106(2):djt431.

9. Erices R, Bravo ML, Gonzalez P, Oliva B, Racordon D, Garrido M, et al. Metformin, at Concentrations Corresponding to the Treatment of Diabetes, Potentiates the Cytotoxic Effects of Carboplatin in Cultures of Ovarian Cancer Cells. Reprod Sci. 2013 Dec 1;20(12):1433-46.

10. Rice MS, Murphy MA, Vitonis AF, Cramer DW, Titus LJ, Tworoger SS, et al. Tubal ligation, hysterectomy and epithelial ovarian cancer in the New England Case-Control Study. Int J Cancer. 2013 Nov 15;133(10):2415-21.

11. Tang L, Lee AH, Su D, Binns CW. Fruit and vegetable consumption associated with reduced risk of epithelial ovarian cancer in southern Chinese women. Gynecol Oncol. 2014 Jan;132(1):241-7.

12. Whilding LM, Archibald KM, Kulbe $H$, Balkwill FR, Öberg $D$, McNeish IA. Vaccinia Virus Induces Programmed Necrosis in Ovarian Cancer Cells. Mol Ther. 2013 Nov;21(11):2074-86.

13. Amor L. F, Vaccaro C. $H$, Martínez N. J, Iturra A. A, U Z, Isabel M. ULTRASONIDO Y CÁNCER DE OVARIO: CARACTERIZACIÓN SUBJETIVA. Rev Chil Obstet Ginecol. 2005;70(5):328-31.

14. Goff BA, Mandel LS, Drescher CW, Urban N, Gough S, Schurman KM, et al. Development of an ovarian cancer symptom index. Cancer. 2007 Jan $15 ; 109(2): 221-7$.

15. Goff BA, Lowe KA, Kane JC, Robertson MD, Gaul MA, Andersen MR. Symptom triggered screening for ovarian cancer: A pilot study of feasibility and acceptability. Gynecol Oncol. 2012 Feb;124(2):230-5.

16. Robyn Andersen M, Goff BA, Lowe KA. Development of an instrument to identify symptoms potentially indicative of ovarian cancer in a primary care clinic setting. Open J Obstet Gynecol. 2012;02(03):183-91. 
17. Hippisley-Cox J, Coupland C. Identifying women with suspected ovarian cancer in primary care: derivation and validation of algorithm. BMJ. 2012 Jan 4;344: d8009.

18. Ovacome. Beta Beat Ovarian Cancer.

19. Arribas M. Diseño y validacion de cuestionarios. Matronas Profesion. $2004 ; 5(17): 23-9$.

20. Dra. Ana Cristina Aranda Flores DDMG. Cancer de Ovario: Diagnostico y Tratamiento [Internet]. Available from: http://www.comego.org.mx/GPC_TextoCompleto/17-Ca\%CC\%81ncer \%20de\%20ovario\%20diagno\%CC\%81stico\%20y\%20tratamiento.pdf

21. Genetics of Breast and Gynecologic Cancers [Internet]. National Cancer Institute. [cited 2015 Oct 5]. Available from: http://www.cancer.gov/types/breast/hp/breast-ovarian-genetics-pdq

22. World Healt Organization. Globocan 2012: Estimated Cancer Incidence, Mortality and Prevalence Worldwide in 2012. [Internet]. Available from: http://globocan.iarc.fr/Pages/fact_sheets_population.aspx 


\section{Anexo I. Instrumento "Diagnostico oportuno de Cáncer de Ovario"}

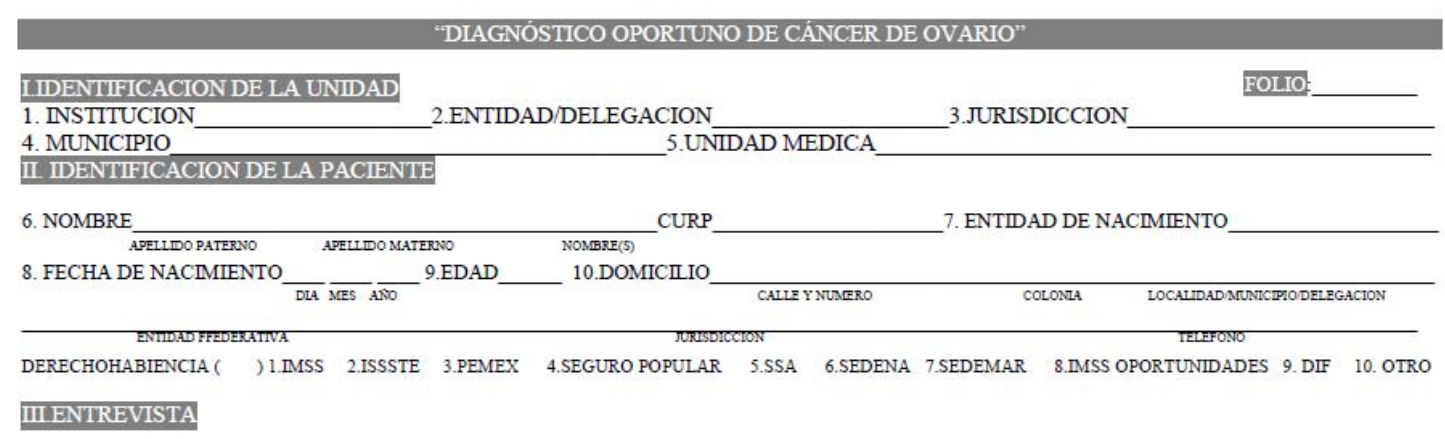

INSTRUCCIONES ¿Ha experimentado alguno de los siguientes sintomas en la actualidad (en el último año)? Indique con una $\mathrm{X}$ su respuesta. En caso de ser afirmativo continúe marcando el número de dias al mes que se presento el sintoma y el tiempo en meses que experimentaste cada sintoma. Para obtener la puntuación multiplicar ambas columnas "dias al mes" y "meses que duro el sintoma". Los resultados válidos por renglón solo serán " 1 " o " 0 ".

ANTECEDENTES: iLe han extirpado los ovarios totalmente (oferectomia )? SI _ NO__ (solo si contesto que NO se realiza el cuestionario).

\begin{tabular}{|c|c|c|c|c|}
\hline Sintomas presentados en la actualidad & Respuesta & $\begin{array}{l}\text { Dias al mes que has } \\
\text { experimentado el } \\
\text { sintoma }\end{array}$ & $\begin{array}{l}\text { Tiempo que duraron los sintomas } \\
\text { en meses. }\end{array}$ & Mńltiplo \\
\hline 1-¿Has experimentando dolor de panza o retorcijones? & $\begin{array}{l}\mathrm{NO}- \\
\mathrm{SI}\end{array}$ & $\begin{array}{l}{ }_{-} \text {De } 1 \text { a } 12 \text { dias }(0) \\
{ }^{13} \text { dias o mas }(1)\end{array}$ & $\begin{array}{cc}\text { Casi nunca } & (0) \\
\text { Menos de } 6 \text { meses } & (0) \\
\text { De } 6 \text { a } 12 \text { meses } & (1) \\
\text { Mayor a } 12 \text { meses } & (0)\end{array}$ & \\
\hline 2-¿Al comer te sientes llena muy rápidamente? & $\begin{array}{l}\mathrm{NO}- \\
\mathrm{SI}\end{array}$ & $\begin{array}{l}{ }_{-}^{\text {De 1a12 dias }(0)} \\
{ }^{13} \text { dias o mas (1) }\end{array}$ & $\begin{array}{cc}\text { Casi nunca } & (0) \\
\text { Menos de } 6 \text { meses } & (0) \\
\text { De } 6 \text { a } 12 \text { meses } & (1) \\
\text { Mayor a } 12 \text { meses } & (0)\end{array}$ & \\
\hline 3-¿Te has sentido inflamada desde que amanece hasta que anoche? & $\begin{array}{l}\mathrm{NO} \\
\mathrm{SI}\end{array}$ & $\begin{array}{l}{ }_{-} \text {De 1a12 dias }(0) \\
{ }^{13} \text { dias o mas (1) }\end{array}$ & $\begin{array}{ll}\text { Casi nunca } & (0) \\
\text { Menos de } 6 \text { meses } & (0) \\
\text { De } 6 \text { a } 12 \text { meses } & (1) \\
\text { Mayor a } 12 \text { meses } & (0)\end{array}$ & \\
\hline $\begin{array}{l}\text { 4-iHas experimentado un aumento en las visitas al baño para hacer } \\
\text { pipi (orinar)? }\end{array}$ & $\mathrm{NO}_{\text {SI }}$ & $\begin{array}{l}\text { _ De 1a12 dias }(0)^{13 \text { dias o mas (1) }}\end{array}$ & $\begin{array}{ll}\text { Casi nunca } & (0) \\
\text { Menos de } 6 \text { meses } & (0) \\
\text { De } 6 \text { a } 12 \text { meses } & (1) \\
\text { Mayor a } 12 \text { meses } & (0)\end{array}$ & \\
\hline $\begin{array}{l}\text { 5-iAlgún integrante de su familia ya sea madre, padre, hermanos de } \\
\text { sangre han padecido cáncer de colon, cáncer de mama, o de ovario? } \\
\text { En esta pregunta solo responda si o no dependiendo su caso. }\end{array}$ & SI__ & No__ $(0)$ & & \\
\hline 6-¿Usted padece o padeció cáncer de mama o colon? & SI__ & No___ (0) & & \\
\hline
\end{tabular}

SI LA PUNTUACIÓN ES MAYOR DE 1, SE REQUERIRA DE QUE SE REALICE UN ESTUDIO DE SANGRE DENOMINADO CA 125, EL ESTUDIO SERÁ GRATUITO Y SE LE ENTREGARA LA SOLICITUD EN ESTE MOMENTO.

IV.SOLOLICITUD DE LABORATORIO

NOMBRE $\mathrm{EDAD}$

UNIDAD QUE REFIERE

FAVOR DE REALIZAR LA PRUEBA DE CA 125 CON FINES DE DESCARTAR POSIBLE LESION EN OVARIO

Acudir en AYUNAS de 8 hrs al LABORATORIO de:

MEDICO QUE ENVIA 